\section{East/Central/South African Genotype in a Chikungunya Outbreak, Dhaka, Bangladesh, 2017}

\author{
Mizanur Rahman, Junya Yamagishi, \\ Rummana Rahim, Abu Hasan, Abu Sobhan
}

Author affiliation: Apollo Hospitals Dhaka, Dhaka, Bangladesh

(M. Rahman, R. Rahim, A. Hasan, A. Sobhan); Hokkaido

University, Sapporo, Japan (J. Yamagishi)

DOI: https://doi.org/10.3201/eid2502.180188

In 2017, an unprecedented increase in febrile illness was observed in Dhaka, Bangladesh. Real-time reverse transcription PCR confirmed that $603(40.2 \%)$ of 1,500 cases were chikungunya fever. Phylogenetic analysis revealed circulation of the non-A226V East/Central/South African genotype of chikungunya virus in Bangladesh.

$\mathrm{C}$ hikungunya fever is a mosquito-transmitted viral disease caused by chikungunya virus (CHIKV; genus $\mathrm{Al}$ phavirus, Togaviridae). Since first isolation from a febrile patient in Tanzania in 1952, CHIKV has been responsible for numerous well-documented outbreaks and epidemics in Africa and Southeast Asia, involving hundreds of thousands of cases (1).

CHIKV strains are clustered into 3 separable genotypes: West African, East/Central/South African (ECSA), and Asian. During 1960-1999, outbreaks in Thailand, Cambodia, Vietnam, Myanmar, the Philippines, Malaysia, Indonesia, Pakistan, and India were caused by strains of the Asian genotype (1). However, since 2005, massive epidemics in the Indian Ocean islands and the worldwide increase in travel have altered circulating genotypic distribution of CHIKV. Studies have shown that different lineages of CHIKV epidemic strains of the ECSA genotype have expanded locally and spread to new areas in Africa, Europe, Asia, and the Americas, in addition to Africa and Asia $(2,3)$. Dengue virus (DENV) is another important arbovirus, spread by the bite of the same group of mosquitoes, Aedes aegypti and Ae. Albopictus, that transmit CHIKV. DENV and CHIKV are often found co-circulating during outbreaks and have an overlapping clinical presentation; thus, misdiagnosis and underreporting of chikungunya infection in dengue-endemic areas is common (4).

In Bangladesh, dengue fever was reported in the mid1960s and dengue hemorrhagic fever in 2000; both diseases are now endemic to Bangladesh (5). In contrast, CHIKV infection in Bangladesh was serologically confirmed for the first time in 2008 (6). Thereafter, sporadic cases of chikungunya have been reported (7); however, in 2017, chikungunya emerged as an important public health issue (8).

Most patients seek healthcare during the acute, febrile phase of the disease, when IgG and IgM titers are typically below the level of detection limits of serologic diagnostic approaches. Therefore, molecular methodologies to detect viral RNA are highly advantageous to detect and differentiate between co-circulating arboviruses and thus facilitate rapid diagnosis and appropriate treatment.

During June 29-October 31, 2017, a total of 1,500 patients visited Apollo Hospitals Dhaka (Dhaka, Bangladesh) with acute onset of fever (days 1-7 from onset), myalgia, arthralgia, and headache; some patients experienced a maculopapular rash, gastrointestinal symptoms, or both. We collected serum samples from these patients for routine serologic testing and for molecular detection of CHIKV and DENV by real-time reverse transcription PCR (rRT-PCR). We extracted RNA from serum by using QIAamp MinElute Virus Spin Kit (QIAGEN, https://www.qiagen.com) according to the manufacturer's instructions. We used purified RNA in a 1-step multiplex rRT-PCR test for the simultaneous detection and differentiation of CHIKV and DENV (Fast Track Diagnostics, http://www.fast-trackdiagnostics.com) on the Rotor Gene Q platform (QIAGEN). Among the 1,500 acute-phase serum samples, rRT-PCR confirmed $603(40.2 \%)$ as CHIKV positive, $233(15.73 \%)$ as DENV positive, and $10(0.66 \%)$ as CHIKV and DENV positive (Appendix Table, https://wwwnc. cdc.gov/EID/article/25/2/18-0188-App1.pdf).

Because of heightened public awareness and government efforts taken to control mosquitoes, the chikungunya cases gradually decreased (Appendix Figure). We then set out to determine the genotype causing the massive CHIKV outbreak.

By using stored RNA, serum samples, or both from deidentified samples, we performed a 1-step rRT-PCR with the SuperScript III 1-step rRT-PCR system with platinum Taq DNA polymerase (ThermoFisher, https://www.thermofisher.com) and a published oligonucleotide primer set (20F and 20R) targeting the E1 gene (9). We randomly selected high-titer viral RNA samples from 32 rRT-PCR-positive samples with a cycle threshold $<30$. We verified PCR products of correct molecular weight by gel electrophoresis and directly sequenced 4 randomly selected amplicons by using Sanger method. We submitted these sequences to DDBJ (accession nos. LC364266-LC364269).

In parallel, we added custom index adaptors with a second rRT-PCR test and sequenced them by using MinION sequencer with the R9.5 flowcell (FLO-107) and 1D2 sequencing kit (LSQ-308) (Nanopore Technologies, https://nanoporetech.com), following the manufacturer's protocol. We determined the consensus sequences of the E1 gene for $27 \mathrm{CHIKV}$ samples with enough reads by using the Canu assembler, even though a couple of inconsistencies were noted between the Sanger and MinION sequences, 


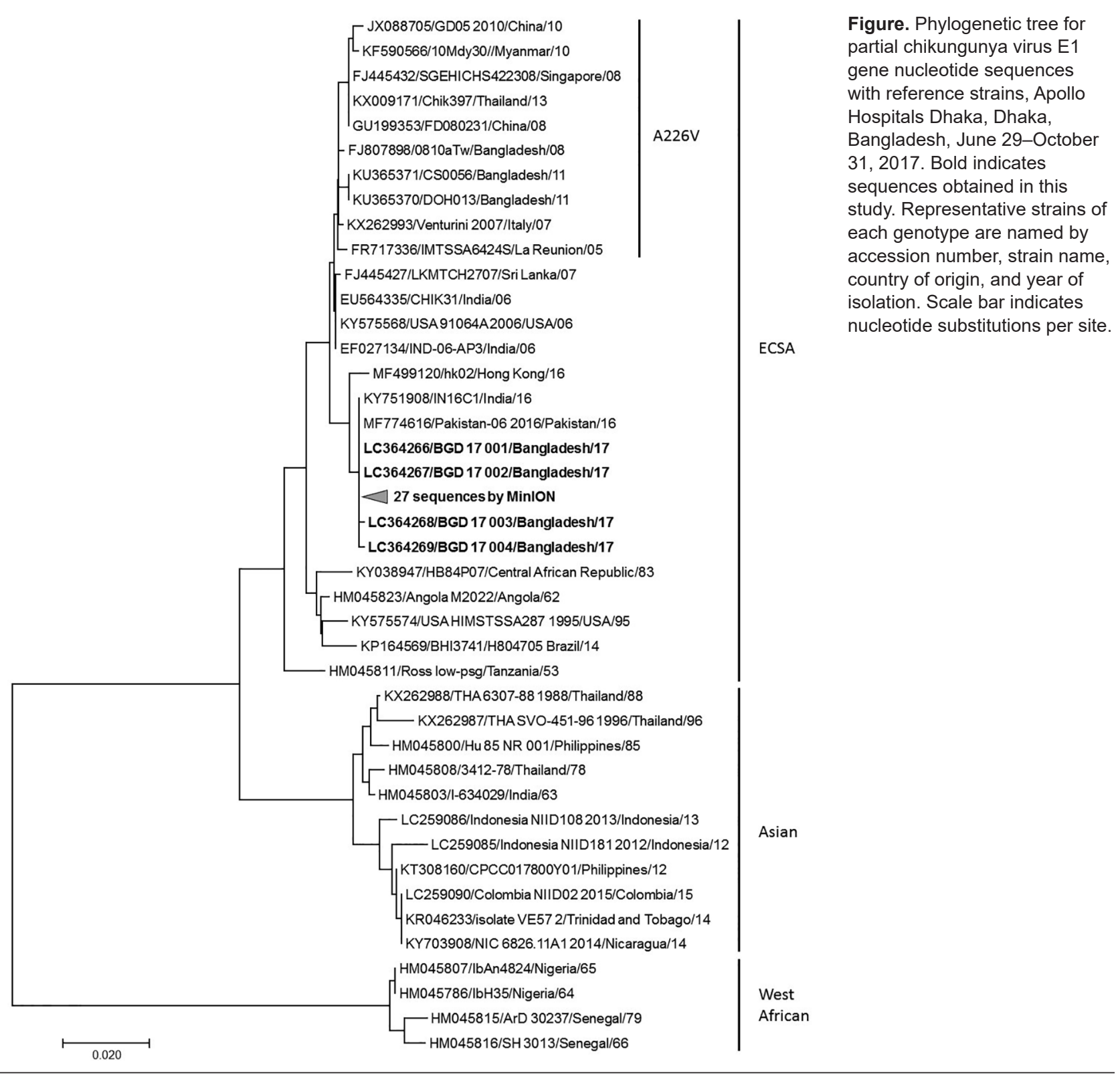

which probably were attributable to the lower sequencing accuracy of the MinION sequencer. We aligned the sequences obtained with the reference strains available from GenBank and constructed a phylogenetic tree (Figure) by using MEGA7 (https://www.megasoftware.net).

The results suggested that all the CHIKV E1 sequences obtained from Bangladesh patients in 2017 belonged to the ECSA genotype and did not harbor the A226V mutation, which is different from the previously reported CHIKV strain detected in Bangladesh in 2008 (10) and 2011 and more genetically related to the lineage circulating endemically in India and Pakistan in 2016. An extensive genome analysis of recent strains of CHIKV along with research on environmental factors is necessary to better understand the factors underlying the recent outbreak to inform efforts to mitigate potential outbreaks in the future.

\section{Acknowledgment}

We thank Junya Yamagishi for his support in sequencing.

This study was approved by the research and ethics committee of Apollo Hospitals Dhaka (ERC 16/2017-1).

\section{About the Author}

Dr. Rahman is a senior consultant at Molecular Diagnostics of Apollo Hospitals Dhaka, Bangladesh. His field of interest was cytokine research, and his current research interest is molecular characterization of arboviruses in Bangladesh. 


\section{References}

1. Powers $\mathrm{AM}$, Logue $\mathrm{CH}$. Changing patterns of chikungunya virus: re-emergence of a zoonotic arbovirus. J Gen Virol. 2007;88:236377. http://dx.doi.org/10.1099/vir.0.82858-0

2. Schuffenecker I, Iteman I, Michault A, Murri S, Frangeul L, Vaney MC, et al. Genome microevolution of chikungunya viruses causing the Indian Ocean outbreak. PLoS Med. 2006;3:e263. http://dx.doi.org/10.1371/journal.pmed.0030263

3. Leparc-Goffart I, Nougairede A, Cassadou S, Prat C, de Lamballerie X. Chikungunya in the Americas. Lancet. 2014; 383:514. http://dx.doi.org/10.1016/S0140-6736(14)60185-9

4. Chahar HS, Bharaj P, Dar L, Guleria R, Kabra SK, Broor S. Co-infections with chikungunya virus and dengue virus in Delhi, India. Emerg Infect Dis. 2009;15:1077-80. http://dx.doi.org/ 10.3201/eid1507.080638

5. icddr,b. Seroprevalence of dengue virus infection in Dhaka, Bangladesh, 2012. Health Sci Bull. 2014;2:1-6.

6. icddr,b. First identified outbreak of chikungunya in Bangladesh, 2008. Health Sci Bull. 2009;7:1-6.

7. Khatun S, Chakraborty A, Rahman M, Nasreen Banu N, Rahman MM, Hasan SMM, et al. An outbreak of chikungunya in rural Bangladesh, 2011. PLoS Neg1 Trop Dis. 2015;9:e0003907. http://dx.doi.org/10.1371/journal.pntd.0003907

8. Kabir I, Dhimal M, Müller R, Banik S, Haque U. The 2017 Dhaka chikungunya outbreak. Lancet Infect Dis. 2017;17:1118. http://dx.doi.org/10.1016/S1473-3099(17)30564-9

9. Sam IC, Loong SK, Michael JC, Chua CL, Wan Sulaiman WY, Vythilingam I, et al. Genotypic and phenotypic characterization of chikungunya virus of different genotypes from Malaysia. PLoS One. 2012;7:e50476. http://dx.doi.org/10.1371/journal.pone.0050476

10. Huang JH, Yang CF, Su CL, Chang SF, Cheng CH, Yu SK, et al. Imported chikungunya virus strains, Taiwan, 2006-2009. Emerg Infect Dis. 2009;15:1854-6. http://dx.doi.org/10.3201/eid1511.090398

Address for correspondence: Mizanur Rahman, Molecular Diagnostics, Apollo Hospitals Dhaka, Plot-81, Block-E, Bashundhara, Dhaka-1229, Bangladesh; email: mizanur.rahman@apollodhaka.com

\section{Dolphin Morbillivirus in Eurasian Otters, Italy}

\section{Iolanda Padalino, Giovanni Di Guardo, Antonio Carbone, Pasquale Troiano, Antonio Parisi, Domenico Galante, Maria Assunta Cafiero, Marta Caruso, Lucia Palazzo, Laura Guarino, Laura De Riso, Cinzia Centelleghe, Sandro Mazzariol, Antonio Petrella}

DOI: https://doi.org/10.3201/eid2502.180256

Author affiliations: Istituto Zooprofilattico Sperimentale della Puglia e della Basilicata, Foggia, Italy (I. Padalino, A. Carbone,

P. Troiano, A. Parisi, D. Galante, M.A. Cafiero, A. Petrella);
University of Teramo, Teramo, Italy (G. Di Guardo); Istituto Zooprofilattico Sperimentale della Puglia e della Basilicata, Matera, Italy (M. Caruso); Istituto Zooprofilattico Sperimentale della Puglia e della Basilicata, Potenza, Italy (L. Palazzo); Istituto Zooprofilattico Sperimentale della Puglia e della Basilicata, Taranto, Italy (L. Guarino); Ente Parco Nazionale del Cilento, Salerno, Italy (L. De Riso); University of Padua, Padua, Italy (C. Centelleghe, S. Mazzariol)

We report biomolecular evidence of dolphin morbillivirus in 4 wild Eurasian otters (Lutra lutra) from southern Italy; 2 animals showed simultaneous immunohistochemical reactivity against morbilliviral antigen. These cases add further concern and support to the progressively expanding host range of dolphin morbillivirus in the western Mediterranean Sea.

$\mathrm{T}$ he genus Morbillivirus comprises several lympho-epithelio-neurotropic, highly pathogenic RNA viruses of domestic and wild vertebrates, including aquatic mammals. Among them, cetacean morbillivirus (CeMV) has been responsible since the 1980s for dramatic epidemics in freeranging cetaceans worldwide (1). Specifically, the CeMV strain termed dolphin morbillivirus (DMV) has caused at least 4 unusual mortality events (UMEs) among striped dolphins (Stenella coeruleoalba) in the western Mediterranean Sea and, to a lesser extent, long-finned pilot whales (Globicephala melas) and other wild cetaceans from the same region (1-3).

We report evidence of DMV infection in 4 wild Eurasian otters (Lutra lutra) from southern Italy (Apulia and Basilicata regions). The animals, all adult females, belonged to a group of 7 individuals found dead at Parco Nazionale del Cilento, a large national park that extends to the coastline of southwestern Italy. The animals underwent necropsy at Istituto Zooprofilattico Sperimentale della Puglia e della Basilicata (Foggia, Italy) during 2016 and 2017, according to an official agreement between the institute and the park aimed at assessing the health and conservation status of the otter population.

Within a multidisciplinary approach framework, we conducted in-depth histopathologic, microbiologic, parasitologic, and ecotoxicologic analyses on the 7 otters, along with biomolecular (reverse transcription PCR [RTPCR]) and immunohistochemical (IHC) investigations for Morbillivirus spp. After using a technique amplifying a highly conserved fragment of the Morbillivirus nucleoprotein (NP) gene (4), we applied 2 additional methods aimed at detecting DMV-specific hemagglutinin (HA) (5) and NP gene sequences (6) for more detailed analysis. To increase the biomolecular results' reliability, we performed all the extraction, amplification, and sequencing steps in 3 different laboratories. We also conducted the histopathological and IHC analyses in 3 different 